\title{
ENERgY HARVESTING ACTIVE NetwORKED TAGS (ENHANTS) FOR UBIQUITOUS OBJECT NetWORKING
}

\section{Maria Gorlatova, Peter Kinget, loannis Kymissis, Dan Rubenstein, Xiaodong Wang, AND GIL ZUSSMAN, COLUMBIA UNIVERSITY}

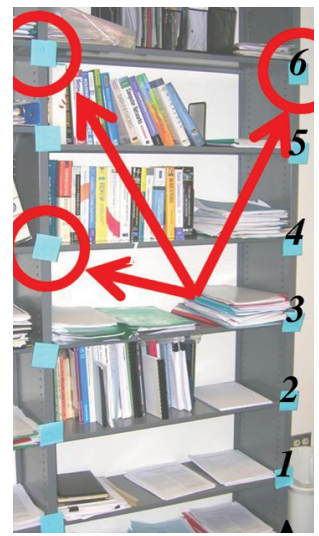

The authors describe paradigm shifts associated with technologies that enable EnHANTs and demonstrate their implications on higher-layer protocols.

\section{ABSTRACT}

This article presents the design challenges posed by a new class of ultra-low-power devices referred to as Energy-Harvesting Active Networked Tags (EnHANTs). EnHANTs are small, flexible, and self-reliant (in terms of energy) devices that can be attached to objects that are traditionally not networked (e.g., books, furniture, walls, doors, toys, keys, produce, and clothing). EnHANTs will enable the Internet of Things by providing the infrastructure for various novel tracking applications. Examples of these applications include locating misplaced items, continuous monitoring of objects, and determining locations of disaster survivors. Recent advances in ultra-low-power circuit design, ultra-wideband (UWB) wireless communications, and organic energy harvesting techniques will enable the realization of EnHANTs in the near future. The harvesting components and the ultra-low-power physical layer have special characteristics whose implications on the higher layers have yet to be studied (e.g., when using UWB communications, the energy required to receive a bit is significantly higher than the energy required to transmit a bit). In this article, we describe paradigm shifts associated with technologies that enable EnHANTs and demonstrate their implications on higher-layer protocols. Moreover, we describe some of the components we have designed for EnHANTs. Finally, we briefly discuss our indoor light measurements and their implications on the design of higher-layer protocols.

\section{INTRODUCTION}

This article focuses on the design challenges posed by a new class of ultra-low-power devices - Energy-Harvesting Active Networked Tags (EnHANTs). EnHANTs are small, flexible, and self-reliant (in terms of energy) devices that can be attached to objects that are traditionally not networked. The realization of EnHANTs is based on recent advances in the areas of solar and piezoelectric energy harvesting [2] and in particular in the area of organic semiconductors that allow having flexible solar cells [3], thereby enabling pervasive use of tags. Another enabler is the development of short-range ultra-lowpower wireless communications circuits, in particular for ultra-wideband (UWB) communications [4].

Following the transition from barcodes to RFIDs, we envision a future transition from RFIDs to EnHANTs that:

- Network: Actively communicate with one another and with EnHANT-friendly devices in order to forward information over a multihop network.

- Operate at ultra-low-power: Spend a few nanoJoules or less on every communicated bit.

- Harvest environmental energy: Collect and store energy from sources such as light, motion, and temperature gradients.

- Are energy adaptive: Alter communications and networking to satisfy energy and harvesting constraints.

- Exchange small messages: Exchange limited information (basically IDs) using low data rates.

- Transmit to short ranges: Communicate only when in close proximity ( 1 to 10 meters) to one another.

- Are thin, flexible, and small (a few square centimeters at most).

As shown in Fig. 1, in terms of complexity, throughput, size, and energy requirements, EnHANTs fit between RFIDs and sensor networks. Similarly to RFIDs, the tags can be affixed to commonplace objects. Presence of power sources and distributed multi-hop operation shift EnHANTs closer to sensor networks than to RFIDs that are traditionally passive and not networked. Compared to sensor nodes, however, EnHANTs operate at significantly lower data rates, consume less energy, and transmit mostly ID information.

EnHANTs will be enablers for the Internet of Things and as such will support a variety of tracking and monitoring applications beyond 


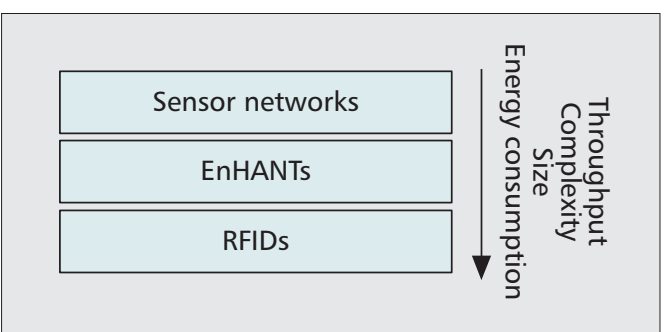

Figure 1. EnHANTs in comparison to Sensor Networks and RFIDs.

what RFIDs permit. While RFIDs make it possible to identify an object in proximity to a reader, EnHANTs will make it possible to search for an object in a network of devices, and to continuously track objects' whereabouts and their proximity to each other. Unlike RFIDs that are activated when placed near a reader, and report only on themselves, EnHANTs can operate continuously, can achieve pervasive coverage due to their networking capabilities, and can report on themselves and other EnHANTs around them.

One application that we plan to demonstrate in the near future is a misplaced library book locator. ${ }^{1}$ The initial prototype will enable library books to identify those among themselves that are significantly misplaced (e.g., in an incorrect section), and report the misplacement. To accomplish this task, each book is assigned a unique ID using an assignment scheme closely related to the Dewey Decimal Classification. Each book has a solar powered tag whose power output is sufficient to transmit and receive information within a radius of one meter or less, and to perform some basic processing. Nearby books wirelessly exchange IDs, and IDs of books that appear out of place are further forwarded through the network of books, eventually propagating to sink nodes. A long term objective could be to determine book locations when books are arbitrarily ordered and to propagate that information to a central server using the multihop wireless network.

The same building blocks used in the library application can enable several other applications. A large variety of items can be tracked and a range of possible desirable or undesirable configurations of objects can be queried for, and can trigger reports. Examples include finding items with particular characteristics in a store, continuous peer monitoring of merchandise in transit, locating misplaced items (e.g., keys or eyeglasses), and locating survivors in disasters such as structural collapse.

EnHANTs are brought about by the recent advances in ultra-low-power communications and energy harvesting technologies. However, current RF transceiver designs, communication and networking protocols, and energy harvesting and management techniques have been developed in isolation and are inadequate for the envisioned EnHANTs applications. Although networking protocols for energy harvesting nodes recently gained attention (e.g., [6-8]), the cross layer interactions between circuit design, energy harvesting, communications, and networking have not been studied in depth. Hence, in this article, we outline the cross-layer design challenges that are posed by this new technology, and describe some of the components we have developed. Moreover, we briefly provide results of our indoor light measurement study and discuss their implications on the design of protocols.

This article is organized as follows. First, we describe related work. Then we discuss energy harvesting and energy storage techniques, as well as ultra-low-power EnHANT communications. We also discuss EnHANT networking as a largescale optimization problem. Finally, we briefly describe an EnHANTs testbed and presents some results of our indoor light measurements.

\section{RELATED WORK}

While the idea of pervasive networks of objects has been proposed before, the harvesting and communications technologies have recently reached a point where networked energetically self-reliant tags are becoming feasible. EnHANTs are likely to be implemented by combining flexible electronics technologies (a.k.a. organic electronics) with CMOS chips supporting Impulse-Radio UWB. By using ImpulseRadio UWB, data is encoded by very short pulses (at the order of nano-seconds) and the energy consumption is very low. Several MAC protocols for IR-UWB sensor networks have been proposed, including the IEEE 802.15.4a standard which inherits many of the IEEE 802.15.4 (ZigBee) functionalities. However, the overhead to provide ranging, high data rates (up to $26 \mathrm{Mb} / \mathrm{s}$ ), and backward compatibility will lead to energy consumption largely exceeding the energy anticipated to be available to EnHANTs.

Energy efficiency in wireless networks has long been a subject of research. Energy-efficient routing, for example, can serve as a basis for EnHANTs routing. However, energy harvesting networks have special characteristics and have been considered only recently. A number of solar energy-harvesting based systems have been implemented and deployed (e.g., [9] and references therein). Current deployments are mostly focused on outdoor energy and use much more energy than EnHANTs will have available. For energy-harvesting sensor networks, adaptive duty cycling [6], dynamic activation of wireless sensors [7], and adaptive data rate control [8] are among the exciting research directions.

Numerous characterizations and measurements of outdoor light energy are available. However, to the best of our knowledge, most indoor light measurements have focused on aesthetic and ergonomic attributes of light, rather than on its energy aspects. Indoor light is examined from an energy perspective in [10]. Yet, there is insufficient data regarding the characteristics of energy available to EnHANTs in indoor settings.

\section{Energy Harvesting}

In this section we briefly describe two major harvesting methods and possible energy storage methods, as well as energy-harvesting and storage devices we developed.
While the idea of

pervasive networks

of objects has been

proposed before, the

harvesting and

communications

technologies have

recently reached a

point where

networked

energetically

self-reliant tags are

becoming feasible.
${ }^{1}$ An RFID-based solution (involving a mobile reader) to the problem of locating misplaced library books has been proposed in [5]. 


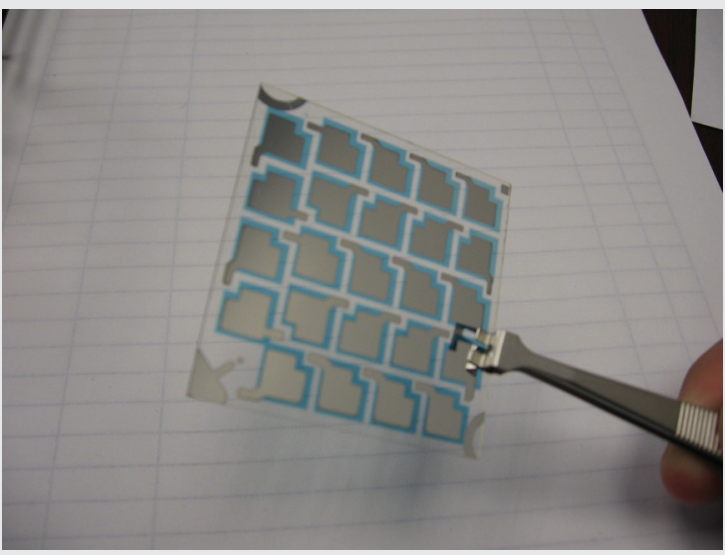

(a)

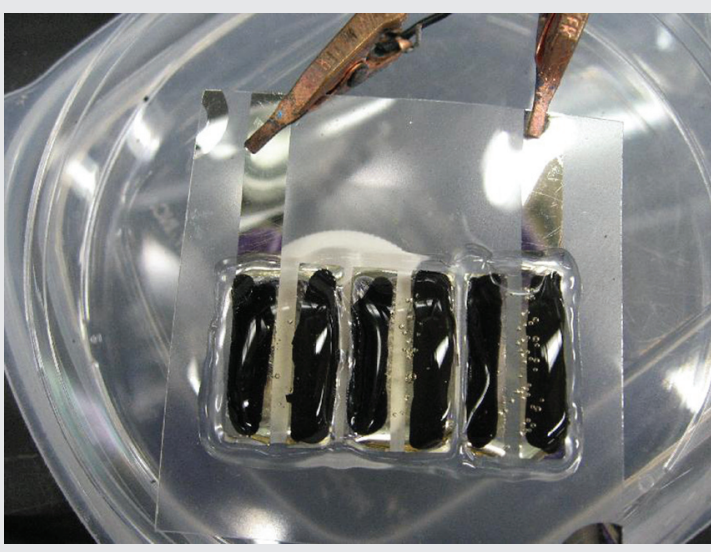

(b)

Figure 2. a) An organic semiconductor-based small-molecule solar cell [12]; (b) a thin-film printable battery, both developed in the Columbia Laboratory for Unconventional Electronics (CLUE).

\section{ENERGY SOURCES}

Many environmental sources of energy are potentially available for harvesting by small devices, i.e., temperature differences, electromagnetic energy, airflow, and vibrations [2]. Here we focus on the most promising harvesting technologies for EnHANTs: solar energy and piezoelectric (motion) harvesting.

Solar Energy Harvesting - Solar energy (light) is one of the most abundant energy sources, with typical irradiance (total energy projected and available for collection) ranging from $100 \mathrm{~mW} / \mathrm{cm}^{2}$ in direct sunlight to $0.1 \mathrm{~mW} / \mathrm{cm}^{2}$ in brightly lit indoor environments (notice the significant difference) [2, 10]. The efficiency of a solar energy harvesting device is defined as the percentage of the available energy that is actually harvested. Conventional single crystal and polycrystalline solar cells, such as those commonly used in calculators, have efficiencies of around 10-20 percent in direct sunlight. However, their efficiency declines with a reduction in energy availability (they are less efficient with dimmer sources), which is important to note due to considerable irradiance difference between direct sunlight and indoor illumination. Conventional solar cells are also inflexible (rigid), which makes it difficult to attach them to non-rigid items such as clothing and paperback books.

An emerging and less explored option is solar energy harvesting based on organic semiconductors [3] (an array of solar cells we recently designed is shown in Fig. 2a). With this technology, solar cells can be made flexible. Moreover, organic semiconductor-based panels operate with constant efficiencies over different brightness levels. However, their efficiency is typically 1-1.5 percent, which is much lower than the efficiency of conventional inorganic solar panels. One of the limitations of organic photovoltaic elements is that they are reactive with water vapor and oxygen. There are several approaches to overcoming this in a flexible, thin film architecture, including encapsulation against environmental degradation. We have demonstrated a flexible, thin film package capable of protecting these solar cells in ambient environments [11]. We have also recently developed a solar cell architecture which uses an organic semiconductor (bis-thienyl pentacene) that has a metastable oxidized state. Even if the device package is penetrated and the material oxidizes, the material returns to its reduced form upon illumination and the cell recovers its performance [12].

To put the energy availability numbers in perspective, consider a system with a $10 \mathrm{~cm}^{2}$ organic semiconductor cell. Outdoors, the system will harvest $10 \mathrm{~cm}^{2} \cdot 100 \mathrm{~mW} / \mathrm{cm}^{2} \cdot 0.01=10 \mathrm{~mW}$. Under the assumption that receiving a bit ${ }^{2}$ requires $1 \mathrm{~nJ}$, the achievable data rate will be $(10$ $\left.\cdot 10^{-3}\right) /\left(1 \cdot 10^{-9}\right)=10 \mathrm{Mb} / \mathrm{s}$. The achievable data rate with indoor lighting will be $\left(10^{-5}\right) /\left(1 \cdot 10^{-9}\right)$ $=10 \mathrm{~kb} / \mathrm{s}$.

Piezoelectric Harvesting - Another potential source of energy is piezoelectric (motion) energy. It can be generated by straining a material (e.g., squeezing or bending flexible items). An example is energy harvesting through footfall, where a harvesting device is placed in a shoe and piezoelectric energy is generated and captured with each step [13].

Piezoelectric harvesting is characterized by the energy captured per actuation at a particular strain (usually 1-3 percent). In [13] it was shown how to harvest $4 \mu \mathrm{J} / \mathrm{cm}^{2}$ per deflection with a strain of approximately 1.5 percent from straining polyvinylidene fluoride (PVDF), a highly compliant piezoelectric polymer. Assume that a $10 \mathrm{~cm}^{2}$ of material is employed in an environment where it is strained once per second (that is, strain frequency of $1 \mathrm{~Hz}$ ). This would provide $10 \cdot 4 \cdot 10^{-6}$ $=40 \mu \mathrm{W}$. If, similar to above, we assume that receiving a bit costs $1 \mathrm{~nJ}$, the bit rate that can be supported is $\left(4.0 \cdot 10^{-5}\right) /\left(1 \cdot 10^{-9}\right)=40 \mathrm{~kb} / \mathrm{s}$.

\section{Energy Storage}

Without the ability to store energy, a device can operate only when directly powered by environmental energy. For a tag, energy storage components need to be compact and efficient, and need to have very low self-discharge rates. 
Rechargeable batteries are an excellent option for energy storage, and numerous battery options are available. Thin film batteries are particularly attractive for EnHANTs since they can be made flexible (a prototype we recently designed is shown in Fig. 2b). Note that a battery needs to be supplied with a voltage exceeding the internal chemical potential in order to start storing provided energy. This implies that charge generated at a low voltage, such as that possibly produced during low harvester excitation (for example, when a solar cell is located in a dimly lit place), cannot be stored without voltage upconversion. However, organic solar cells discussed above can produce relatively high voltage even at low excitation. This highlights another advantage of using organic solar cells for EnHANTs: in a system using an organic solar cell, a battery can store energy when illumination is low, while in a conventional system a battery would not store charge produced at that illumination level.

Capacitors can also be used for energy storage. Capacitors can receive any charge which exceeds their stored voltage and be cycled many more times than batteries. The disadvantage of using capacitors, however, is that as a capacitor gets more charged, it becomes more difficult to add charge, and large electrolytic capacitors selfdischarge over hours or days. The energy density (how much energy can be stored per unit of volume) of capacitors is also much lower. A typical battery can store about $1000 \mathrm{~J} / \mathrm{cm}^{3}$, whereas high performance ceramic capacitors can store 1$10 \mathrm{~J} / \mathrm{cm}^{3}$.

\section{LOW POWER COMMUNICATIONS}

Ultra-wide band (UWB) impulse radio (IR) is a compelling technology for short range ultra-lowpower wireless communications. It uses very short pulses (on the order of nano-seconds) that are transmitted at regular time intervals with the data encoded in the pulse amplitude, phase, frequency, or position. At low data rates, the short duration of the pulses allows most circuitry in the transmitter or receiver to be shut down between pulses, resulting in significant power savings compared to narrow-band systems.

Practical CMOS IR circuits with energy consumption on the order of a nano Joule per bit have recently been demonstrated. For example, in [4] we demonstrate a UWB receiver and transmitter that require $1.65 \mathrm{~nJ} / \mathrm{bit}$ and $280 \mathrm{pJ} / \mathrm{bit}$, respectively, at a data rate of $1 \mathrm{Mb} / \mathrm{s}^{3}{ }^{3}$ Our ongoing research indicates that UWB-IR transceivers in the $3-5 \mathrm{GHz}$ band with data rates of $0.1-1$ $\mathrm{Mb} / \mathrm{s}$ and receiver and transmitter consumption of less than $500 \mathrm{pJ} / \mathrm{bit}$ and $50 \mathrm{pJ} / \mathrm{bit}$, respectively, are within reach.

In this section, we outline the envisioned design of UWB transceivers for EnHANTs, highlight UWB features that are important for higher-layer protocols, and briefly discuss higher layer protocol challenges.

\section{Energy Costs - a Paradigm Shift}

An important paradigm shift associated with ultra-low-power IR-UWB transceivers is that the energy to receive a bit is much higher than the energy to transmit a bit. This is significantly dif- ferent from traditional technologies. In largescale systems the energy to transmit is higher than the energy to receive, due to transmitter's need to generate a signal with enough power to reach long distances. In GSM cellular phones, for example, the transmitter power is on the order of Watts, and the receiver power is on the order of hundreds of $\mathrm{mW}$. In shorter-range systems the energy to transmit and to receive are on the same order of magnitude. For example, for Bluetooth and IEEE 802.15.4, the transmit and the receive power are on the order of tens of $\mathrm{mW}$.

In IR-UWB, transmitter energy consumption is further reduced. In conventional systems, narrow-band modulated sinusoids are transmitted, and the transmitter has to be active for the entire duration of the signal transmission. As mentioned above, in UWB very short pulses convey information, so the transmitter and receiver can wake up for very short time intervals to generate and receive pulses, and can sleep between subsequent pulses. The receiver's energy is mostly spent on running low-noise amplification and data-detection circuits that are consuming energy whenever the device listens to the medium. Hence, there is no difference (in terms of energy) between receiving information and listening to the medium.

The new energy trade-offs call for the design of novel protocols for EnHANTs, since many legacy algorithms were developed under the assumption of transmission being more expensive than reception.

\section{INACCURATE CLOCKS}

Accurate on-chip clocks cannot be powered down and thus consume a lot of energy. Hence, they have to be avoided to achieve ultra-lowpower operation. One viable solution is to use energetically cheap clocks (e.g., clocks available from ultra-low-power ring oscillators). However, the frequency of such clocks will vary significantly from tag to tag and its stability over time is also poor.

A UWB receiver has to wake up at certain times in order to receive pulses. Determining these times with inaccurate clocks imposes major challenges, so while inaccurate clocks save energy, they increase the energy spent on reception. Moreover, traditional low-power sleep-wake protocols heavily rely on the use of accurate time slots. Hence, eliminating the availability of accurate clocks in a tag requires redesigning protocols that were originally designed specifically for energy efficient networking.

\section{Design AlloWing fOR a High-POWer MOde}

EnHANTs transceivers employ numerous design features targeted at decreasing energy consumption as much as possible. However, in some cases it may be beneficial to spend more energy than what is typically spent by a tag (i.e., when the battery is fully charged and the tag is harvesting energy). In such cases an EnHANT can operate in a high-power mode. To enable such modes, EnHANT circuitry can be designed to contain optional power-hungry hardware modules that would allow, for example, for a more accurate/faster clock to be turned on. Other
The new energy trade-offs call for the design of novel protocols for EnHANTs, since many legacy algorithms were developed under the assumption of transmission being more expensive than reception.

\footnotetext{
${ }^{3}$ Energy consumption is measured at a particular data rate, since per-bit parameters differ at different data rates. Note that at lower bit rates, the energy per bit can increase due to the impact of fixed rateindependent circuitry.
} 


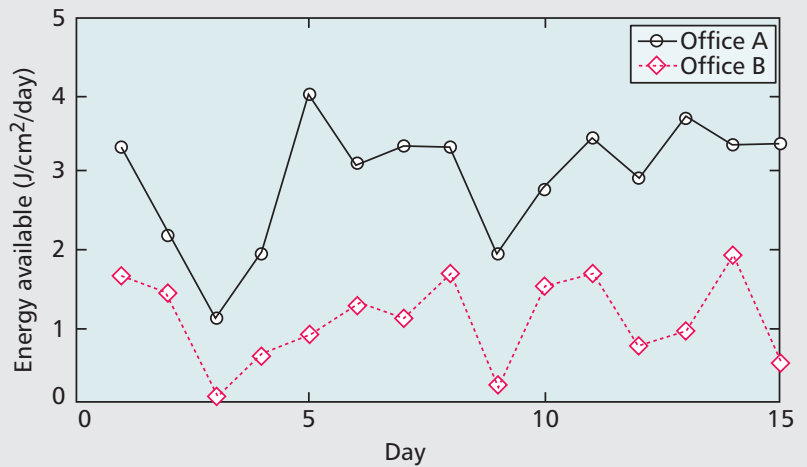

(a)

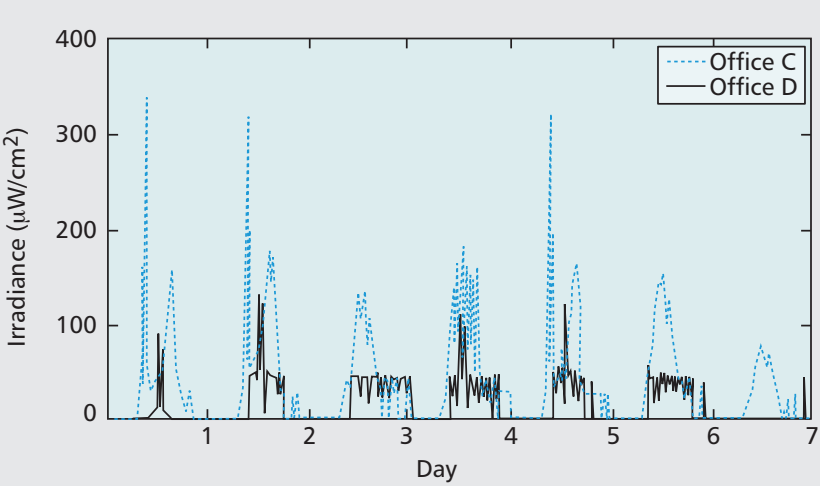

(b)

Figure 3. Indoor energy availability measurements: a) total energy available per day in two different locations over 15 days and b) irradiance in two different locations over 7 days.

components that could be considered are more sensitive receiver stages and more selective filters. Realizing the benefits of high-power modes is an interesting design challenge for higherlayer protocols.

\section{Additional Challenges in EnHANT COMMUNICATIONS}

Similar to traditional sensor networking, in pairwise EnHANT communications three states can be identified: independent (tags not communicating), paired (synchronized and exchanging periodic keep-alive bursts to stay synchronized), and communicating (sending and receiving data). To control its energy spending, a tag can move between states with respect to each of its neighbors. In addition, in each state a tag can spend energy at different rates.

Communicating EnHANTs need to coordinate their transmissions in order to ensure that they do not run out of energy. Note that since listening is energetically expensive, the communication rate of a tag that transmits information is not only related to tag's own energy, but is also tightly coupled with the energy of a tag that receives the information. To make joint decisions on communication rates, EnHANTs need to exchange information about their energy states. However, exchanges of complete energy parameters may be too costly. Determining how much information EnHANTs should exchange and deciding on the frequency of information updates remains an open problem.

Since it is energetically cheaper for a tag to transmit than to listen, if a tag is very low on energy, it could transmit pulses but not listen to the medium. Accommodating the presence of such transmit-only tags is a topic for further research.

A benefit of the harvesting system is that EnHANTs in close proximity will be subject to common stimuli through their energy harvesting channels. Examples include lights turning on/off or running with modulated intensity (e.g., the $60 \mathrm{~Hz}$ variation in fluorescent lighting), or vibrations felt by more than one tag. For instance, when a light is turned on, a tag can assume that the energy parameters of all its neighbors change, and behave accordingly. Information about the relative similarities or differences between EnHANTs energy stimuli can be used for synchronization via a channel which is effectively always open.

Finally, security and privacy are very important issues for the proposed EnHANTs applications. Lightweight techniques that have been designed for sensor networks and for RFIDs can serve as starting points in EnHANTs security research.

\section{EnHANT ENERgy Spending AS A Large-Scale Optimization Problem}

When communicating with each of its neighbors, a tag decides on the state of communication and the rate of energy consumption. When many tags are involved, the joint decisions on states and rates form a large-scale optimization problem, for which a suitable solution needs to be calculated by low-power EnHANTs without extensive exchange of control information.

The complexity of tag's energy harvesting system needs to be captured in a relatively simple way. A possible abstraction is to characterize the energy harvesting system by the maximum energy storage capacity $C$ (Joules), the currently available energy level $E$ (Joules), the energy charge rate $r$ (Watts), and the energy consumption rate $e$ (Watts). Note that the energy charge rate $r$ depends both on the harvesting rate and the properties of the energy storage. For example, when a battery is used, $r$ is positive only when the voltage at the energy harvesting component exceeds the internal chemical potential of the battery. When a capacitor is used, the relationship of $r$ and energy harvesting rate varies with $E$. The $r$ values of different EnHANTs operating in the same environment will be significantly different. Our experiments with commercial hardware show that harvesting rates of identical harvesting devices under identical light conditions can differ by about 30 percent. 


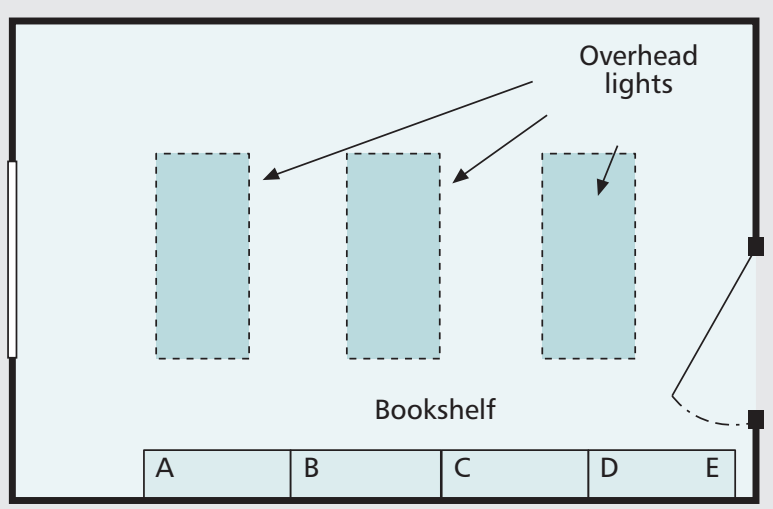

(a)

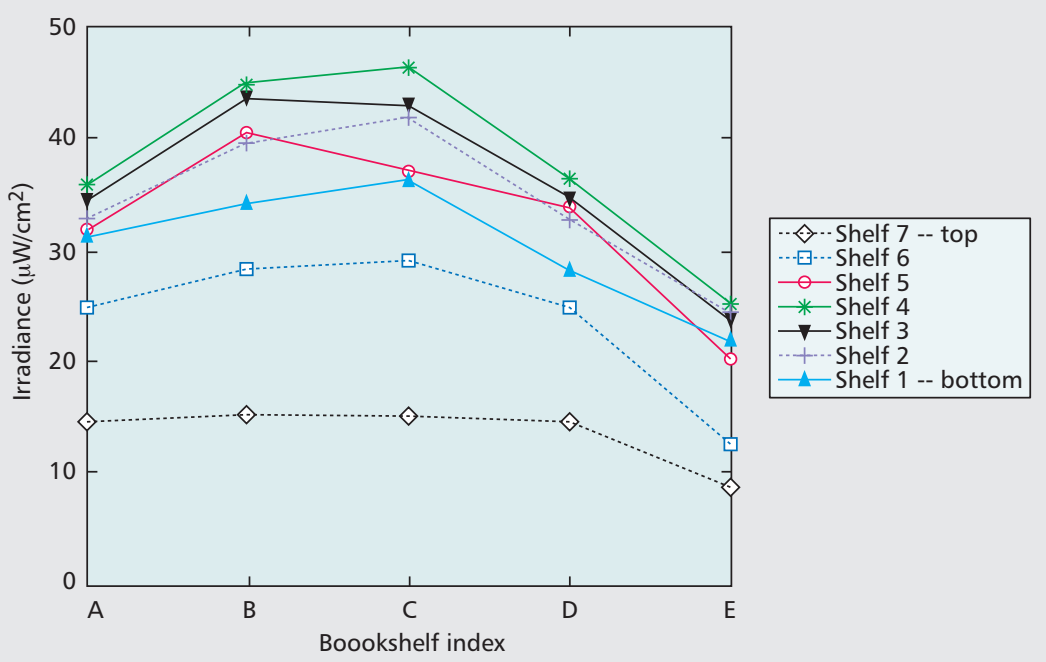

(c)

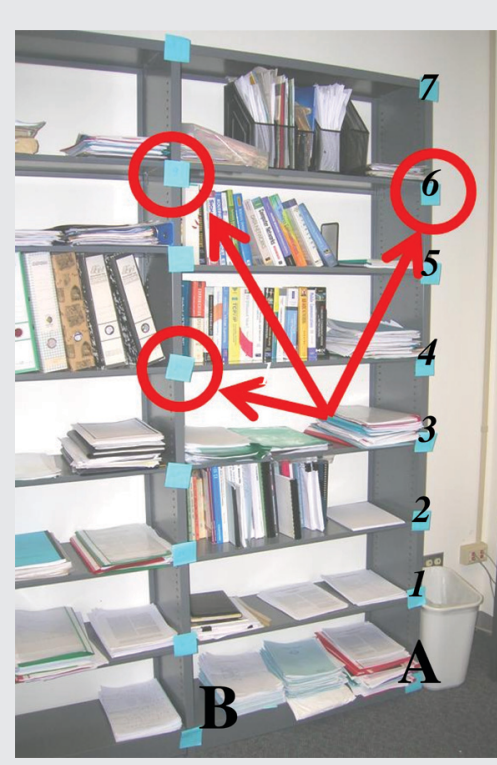

(b)

When communicating with each of its

neighbors, a tag decides on the state of communication and the rate of energy consumption. When many tags are involved, the joint decisions on states and rates form a large-scale optimization problem.

Figure 4. Irradiance measurements in different parts of a bookshelf: a) the floor plan of the office where the measurements were taken; $b$ ) the leftmost stall (stall $A$ ) in which measurement were taken with measurement locations; and c) the irradiance values in different locations on the bookshelf (measurements taken when outdoor light does not affect the values).

Inventory control theory [14] can potentially be used to formulate and solve the optimization problem. An energy-harvesting tag can be viewed as a manufacturing system composed of a factory and a warehouse. Consider the above-identified system parameters $C, r$, $e$, and $E$. A factory-warehouse has a finite capacity, a rate at which products are manufactured, a rate at which products are purchased, and a level of inventory - all of which directly correspond to the harvesting system parameters. A warehouse inventory management system strives to ensure that the demand is met and that there are no shortages. Similarly, the tag's energy management system should ensure that it utilizes the avail- able resources efficiently and does not run out of energy.

The similarities with inventory systems allow for simple modeling of a stand-alone EnHANT. A network of EnHANTs calls for extensions of existing inventory management models. A particular challenge in EnHANTs is the tight coupling of energy spending of different tags. An EnHANT network can be viewed as a network of factories in which the behavior of one significantly affects the behavior of the others. Further, while in a manufacturing system the central controller has complete knowledge, in a network of tags, distributed low complexity algorithms using partial knowledge will have to be employed. 
Existing wireless

mote designs,

mostly optimized for

wireless sensor

applications, do not

permit experimenta-

tion with physical

layer communica-

tions protocols.

Hence, we are in the

process of building

EnHANT prototypes.

\section{Energy HarVesting EXPeriments \& ENHANT TEStbed DeVElopMent}

It is important to quantify how much energy can be harvested indoors, and the spatio-temporal variability. To the best of our knowledge, no extensive long-term indoor light energy measurement studies have been conducted. In this section, we outline the development of an experimental EnHANTs testbed, and briefly discuss some of our energy harvesting measurements.

\section{EnHANT Testbed DeVElopment}

Existing wireless mote designs, mostly optimized for wireless sensor applications, do not permit experimentation with physical layer communications protocols. Hence, we are in the process of building EnHANT prototypes.

Our current first-phase prototypes mostly use commercial off-the-shelf (COTS) communication and energy harvesting hardware components. One current prototype is based on a MICA2 mote attached to a small battery and a custom flexible solar cell [12] that charges the battery, and to a TAOS TSL230rd light-to-frequency converter for light measurements. These prototypes serve as a platform for further custom hardware integration and for experiments on energy harvesting aware communication protocols [15]. We are currently replacing the COTS components with custom-designed hardware, namely, a thin film printable battery similar to the one shown in Fig. 2, and a custom-designed UWB transceiver [4]

\section{INDOOR LIGHT ENERGY MEASUREMENTS}

Since June 2009 we have been conducting a light measurement study in office buildings in New York City. We continuously record irradiance in several different locations using TAOS TSL230rd light-to-frequency converters installed on LabJack U3 Data Acquisition boards. For each location, we calculate from these measurements the total energy available to a $1 \mathrm{~cm}^{2}$ solar cell in a day (denoted by $\left.E_{d}\right) .{ }^{4}$ For example, Fig. 3a shows the values of $E_{d}$ in two different locations over 15 days (Jan. 21, 2010-Feb. 6, 2010). There are significant differences between the energy availabilities at the different locations: on average, the LabJack boards receive $2.9 \mathrm{~J} / \mathrm{cm}^{2} / \mathrm{day}$ and $1.1 \mathrm{~J} / \mathrm{cm}^{2} /$ day in Office A and B, respectively. The difference stems from different office locations and layouts as well as occupants' behaviors (e.g., raising the blinds). Assuming a $10 \mathrm{~cm}^{2}$ solar cell with a 1 percent overall conversion efficiency and a bit communication cost of $1 \mathrm{~nJ} / \mathrm{bit}$, the average achievable data rate is $E_{d} \cdot 10 \mathrm{~cm}^{2}$. $0.01 /\left(24 \cdot 3600 \mathrm{~s} /\right.$ day $\left.\cdot 1 \cdot 10^{-9} \mathrm{~J} / \mathrm{bit}\right)$. Accordingly, the average achievable continuous bit rates are $3.35 \mathrm{~kb} / \mathrm{s}$ for a tag in Office A and $1.27 \mathrm{~kb} / \mathrm{s}$ for a tag in Office B.

As can be seen in Fig. 3a, for a particular location, the available energy also varies greatly between different days (the standard deviations are 0.8 and 0.56 in Office A and B, respectively), due to factors such as weather conditions, indoor lights use, and shading use. In addition to the temporal variations between days, there may be significant temporal variations within a day. The characteristics of these variations depend on the location. For instance, Fig. 3b shows irradiance levels recorded over 7 days (Jan. 31, 2010-Feb. $6,2010)$ in two different offices. The shapes of the two curves are quite different. In Office C, the LabJack board gets most of the energy from sunlight, resulting in variations related to the Sun's diurnal cycle. In Office D, the LabJack board gets most of the energy from artificial light sources and is exposed to direct sunlight for only a short duration. This results in a curve that is flat during most of the work day and has minor temporal variations.

The measurements also indicate that even when there is little temporal variability (e.g., within an office illuminated by artificial sources), there is still high spatial variability. For example, in Fig. 4 we demonstrate how the irradiance levels vary as a function of the distance (and orientation) from light sources. We measured the irradiance levels at different locations on a bookshelf which consists of 4 stalls with 7 shelves in each, and is illuminated by overhead fluorescent lights as shown in Fig. 4a. We took measurements at the intersection points of shelves and bookshelf stalls, as depicted in Fig. 4b. Figure 4c shows the measured irradiance levels for each of the shelves. It demonstrates that the available energy levels are significantly different even within a seemingly uniform environment.

The communications and networking protocols need to be designed such that they can cope with this high spatial variability as well as with the spatial and temporal variations demonstrated in Fig. 3.

\section{CONCLUSIONS}

Ultra-low-power Energy Harvesting Active Networked Tags (EnHANTs) are enablers for a new type of a ubiquitous wireless network that lies in the domain between sensor networks and RFIDs. While RFIDs make it possible to identify an object in proximity to a reader, EnHANTs make it possible to search for an object in a network of devices and continuously monitor objects' locations and proximities to each other. EnHANTs will enable the Internet of Things by providing infrastructure for novel tracking applications.

EnHANTs necessitate rethinking of communications and networking principles, and require careful examination of the particularities of ultra-low-power and energy harvesting technologies. We outlined several important characteristics of EnHANTs and described harvesting and communications devices composing them. We have also described some of our indoor light measurements. We have shown that the nature of EnHANTs requires a cross-layer approach to enable effective networking between devices with severe power and harvesting constraints.

\section{ACKNOWLEDGMENTS}

This work was supported in part by the Vodafone Americas Foundation Wireless Innovation Project, Google Inc., NSF grants CNS-0916263 and CCF-0964497, and by an NSERC CGS 
grant. We thank Enlin Xu, Michael Zapas, and Matthias Bahlke for their assistance with the light measurements.

\section{REFERENCES}

[1] M. Gorlatova et al., "Challenge: ultra-Low-Power Energy-Harvesting Active Networked Tags (EnHANTs)," Proc. ACM MobiCom'09, Sept. 2009.

[2] J. Paradiso and T. Starner, "Energy Scavenging for Mobile and Wireless Electronics," IEEE Pervasive Comp., vol. 4, no. 1, pp. 18-27, 2005.

[3] P. Peumans, A. Yakimov, and S. Forrest, "Small Molecular Weight Organic Thin-Film Photodetectors and Solar Cells," J. Appl. Phys., vol. 93, 2003, p. 3693.

[4] M. Crepaldi et al., "An ultra-Low-Power InterferenceRobust IR-UWB Transceiver Chipset Using Self-Synchronizing OOK Modulation," Proc. IEEE ISSCC'10, Feb. 2010.

[5] I. Ehrenberg, C. Floerkemeier, and S. Sarma, "Inventory Management with an RFID-Equipped Mobile Robot," Proc. IEEE CASE'07, Sept. 2007.

[6] A. Kansal et al., "Power Management in Energy Harvesting Sensor Networks," ACM Trans. Embedded Comp. Sys., vol. 6, no. 4, 2007, p. 32

[7] K. Kar, A. Krishnamurthy, and N. Jaggi, "Dynamic Node Activation in Networks of Rechargeable Sensors," IEEE/ACM Trans. Net., vol. 14, no. 1, 2006, pp. 15-26.

[8] K.-W. Fan, Z. Zheng, and P. Sinha, "Steady and Fair Rate Allocation for Rechargeable Sensors in Perpetual Sensor Networks," Proc. ACM SenSys'08, Nov. 2008.

[9] J. Taneja, J. Jeong, and D. Culler, "Design, Modeling, and Capacity Planning for Micro-Solar Power Sensor Networks," Proc. IEEE IPSN'08, Apr. 2008.

[10] J. Randall, Designing Indoor Solar Products, 1st Ed., Wiley, 2005.

[11] M. E. Bahlke, S. P. Subbarao, and I. Kymissis, "A Laboratory Process for Encapsulation of Air Sensitive Organic Devices," IEEE Trans. Electron Devices, vol. 57, no. 1, Jan. 2010, pp. 153-56.

[12] A. A. Gorodetsky et al., "Solar Cell from a Solution Processable Pentacene with Improved Air Stability," Chem. Mater., vol. 21, no. 18, 2009, pp. 4090-92.

[13] J. Kymissis et al., "Parasitic Power Harvesting in Shoes," Proc. 2nd Int'l. Symp. Wearable Computers, 1998.

[14] D. Simchi-Levi, X. Chen, and J. Bramel, The Logic of Logistics, 2nd Ed., Springer, 2005.

[15] M. Gorlatova et al., "Demo: Prototyping Energy Harvesting Active Networked Tags (EnHANTs) with MICA2 motes," Proc. IEEE SECON'10, June 2010.

\section{BIOGRAPHIES}

MARIA Gorlatova (mag2206@columbia.edu) is a Ph.D. Candidate in the Columbia University Electrical Engineering Department. Her research interests are in networking ultralow-power wireless devices. She has received her B.S. and M.S. degrees from the University of Ottawa, Canada. Prior to joining Columbia's Ph.D. program, Maria worked as a research scientist at Defence R\&D Canada and at Telcordia Technologies. Maria is a recipient of Columbia University Presidential Fellowship and Canadian Graduate Scholar (CGS) NSERC Fellowships.

PETER R. KINGET (kinget@ee.columbia.edu) received an engineering degree in Electrical and Mechanical Engineering and the Ph.D. in electrical engineering from the Katholieke Universiteit Leuven, Belgium. He has worked in industrial research and development at Bell Laboratories, Broadcom, Celight and Multilink before joining the faculty of the Department of Electrical Engineering, Columbia University, in 2002. His research interests are in analog and RF integrated circuits and signal processing using nanoscale CMOS technologies. He has been an Associate Editor of the IEEE Journal of Solid State Circuits (2003-2007) and the IEEE Transactions on Circuits and Systems II (2008-2009). He is also serving on the Technical Program Committees of the International Solid-State Circuits Conference and the European Solid-State Circuits Conference. $\mathrm{He}$ is a "Distinguished Lecturer" for the IEEE Solid-State Circuits Society.

IOANNIS (JOHN) KYMISSIS (johnkym@ee.columbia.edu) is an Assistant Professor of Electrical Engineering at Columbia University. His research focuses on the processing, design, and applications of thin film semiconductors to sensors and actuators, with a particular emphasis on the use of organic semiconductors and recrystallized silicon. John graduated with his SB, M.Eng., and Ph.D. degrees from MIT, and is a senior member of the IEEE.

DAN RUBENSTEIn (danr@cs.columbia.edu) is an Associate Professor in the Department of Computer Science at Columbia University. He received a Ph.D. in Computer Science from University of Massachusetts, Amherst. His research interests are in network technologies, applications, and performance analysis. He is an editor for IEEE/ACM Transactions on Networking, program chair of ACM Sigmetrics 2011, and has received an NSF CAREER Award, IBM Faculty Award, the three paper awards from ACM SIGMETRICS 2000, IEEE ICNP 2003, and ACM CoNext 2008.

XiAOdONG WANG [S'98, M'98, SM'04, F'08] (wangx@ee.columbia.edu) is a Professor of Electrical Engineering at Columbia University. He received the Ph.D. degree in Electrical Engineering from Princeton University. His research interests fall in the general areas of computing, signal processing and communications, and he has published extensively in these areas. Among his publications is a recent book entitled "Wireless Communication Systems: Advanced Techniques for Signal Reception", published by Prentice Hall in 2003. He received the 1999 NSF CAREER Award, and the 2001 IEEE Communications Society and Information Theory Society Joint Paper Award. He has served as an Associate Editor for the IEEE Transactions on Communications, the IEEE Transactions on Wireless Communications, the IEEE Transactions on Signal Processing, and the IEEE Transactions on Information Theory. He is a Fellow of the IEEE and listed as an ISI Highly-cited Author.

GIL ZUSSMAN received his Ph.D. degree in electrical engineering from the Technion in 2004. Between 2004 and 2007 he was a postdoctoral associate at MIT. He is an assistant professor of electrical engineering at Columbia University, and his research interests are in the area of wireless networks. He is a recipient of the Fulbright and the Marie Curie Outgoing International Fellowships, the IFIP Networking 2002 Best Student Paper Award, the ACM SIGMETRICS '06 Best Paper Award, and the DTRA Young Investigator Award.
While RFIDs make it

possible to identify

an object in

proximity to a

reader, EnHANTS

make it possible to

search for an object

in a network of

devices and

continuously monitor

objects' locations

and proximities to

each other. 